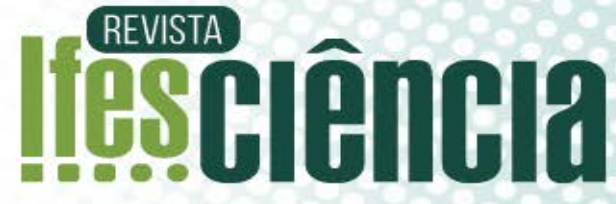

\section{BIOCARVÃo E ÁGUA RESIDUÁRIA DE SUINOCULTURA NO DESENVOLVIMENTO INICIAL DO CAFÉ CONILON}

\author{
BIOCHAR AND PIG WASTE WATER IN THE INITIAL DEVELOPMENT OF CONILON \\ COFFEE CONILON \\ ${ }^{1}$ Leidiane de Souza Azevedo \\ ${ }^{3}$ Mayara Cazadini Carlos \\ ${ }^{1}$ Alex Justino Zacarias \\ ${ }^{2}$ Rebyson Bissaco Guidinelle \\ ${ }^{1}$ Otacílio José Passos Rangel \\ ${ }^{2}$ Renato Ribeiro Passos
}

\begin{abstract}
${ }^{1}$ Instituto Federal do Espírito Santo - Campus de Alegre, Rodovia ES-482, Km 47 Distrito de Rive - Caixa Postal 47 | Alegre - ES | CEP: 29500-000: leidiazevedo98@gmail.com; alexjustino12@gmail.com; ojprangel@ifes.edu.br

${ }^{2}$ Universidade Federal do Espírito Santo - Campus de Alegre, Alto Universitário, s/n ${ }^{\circ}$ - Guararema, Alegre - ES

| CEP 29500-000: rebysonguidinelle@gmail.com; renatoribeiropassos@hotmail.com; ${ }^{3}$ Universidade Estadual Norte Fluminense Darcy Ribeiro, Centro de Ciências e Tecnologias Agropecuárias, Avenida Alberto Lamego, 2000 - Parque Califórnia - Campos dos Goytacazes, RJ, CEP 28013-602. E-mail: may_cazadini@hotmail.com
\end{abstract}

Artigo submetido em 30/10/2020, aceito em 09/11/2020 e publicado em 23/12/2020.

Resumo: O objetivo do estudo foi avaliar o efeito de diferentes doses de biocarvão, proveniente da palha de café conilon, associado com água residuária de suinocultura no desenvolvimento de plantas de café conilon. O experimento foi conduzido em casa de vegetação localizada no Instituto Federal do Espirito Santo (Ifes) - Campus de Alegre. Os tratamentos consistiram da combinação de cinco doses de biocarvão de palha de café conilon (0, 5, 10, 15 e 20 t/ha), com duas águas de irrigação (água residuária de suinocultura-ARS e água bruta- $\mathrm{AB}$ ). Para a obtenção do biocarvão, a palha de café foi acondicionada em um reator de pirólise e carbonizado a $350^{\circ} \mathrm{C}$. A ARS foi proveniente do processo de biodigestão de resíduos de suinocultura e a $\mathrm{AB}$ foi originária da água de abastecimento sem tratamento. O solo utilizado foi um Latossolo vermelho-amarelo, coletado na profundidade $0-40 \mathrm{~cm}$. O experimento foi conduzido por 150 dias, quando foram avaliados parâmetros morfoagronômicos de desenvolvimento do cafeeiro. As doses de biocarvão não influenciaram no desenvolvimento de plantas até os 150 dias de avaliação. A irrigação com ARS promoveu melhores resultados de altura de planta, número de ramos plagiotrópicos e massa da matéria seca da parte aérea das plantas de café conilon.

Palavras-chave: Coffea canephora; palha de café; resíduo de suinocultura.

Abstract: The aim of the study was to evaluate the effect of different doses of biochar, derived from conilon coffee straw, associated with swine wastewater, on the development of conilon coffee plants. The experiment was conducted in a greenhouse located at the Federal Institute of Espirito Santo (Ifes) - Campus de Alegre. The treatments consisted of the combination of five doses of biofuel from conilon coffee straw $(0,5,10,15$ and $20 \mathrm{t} / \mathrm{ha})$, with two irrigation waters (swine waste water-SWW and raw water-AB). To obtain the biochar, the coffee straw was stored in a pyrolysis reactor and carbonized at $350^{\circ} \mathrm{C}$. The SWW came from the pig digestion biodigestion process and the $\mathrm{AB}$ 
originated from the untreated supply water. The soil used was a red-yellow Latosol, collected at a depth of 0-40 cm. The experiment was conducted for 150 days, when morpho-agronomic parameters of coffee development were evaluated. The doses of biochar did not influence the development of plants until the 150 days of evaluation. The irrigation with SWW promoted better results of plant height, number of plagiotropic branches and dry matter weight of the aerial part of conilon coffee plants.

Keywords: Coffea canephora; coffee straw; pig farming residue.

\section{INTRODUÇÃO}

Diante do aumento da população mundial, da demanda por alimentos, com o provável aumento na pressão sobre os recursos naturais e consequentemente intensificação da degradação do planeta, pesquisadores do mundo inteiro têm realizado trabalhos visando o desenvolvimento de uma agricultura de baixo impacto ambiental. Um dos preceitos desse modelo de produção é o aproveitamento sustentável de todos recursos presentes na propriedade rural, usando o mínimo possível de insumos externos como fertilizantes, herbicidas e inseticidas.

Biocarvão, do inglês biochar, é o produto oriundo da decomposição térmica de materiais orgânicos sob baixa disponibilidade de oxigênio e temperaturas que podem variar entre 350 a $700^{\circ} \mathrm{C}$ (SHACKLEY et al., 2012; SRINIVASAN et al., 2015).

A alta concentração de carbono nos biocarvões é um dos fatores que mais chamam a atenção dos pesquisadores para utilização desse material como condicionador de solos (OLIVEIRA et al., 2017; EL-NAGGAR et al., 2019; MAROUŠEK et al., 2019; FONSECA et al., 2020). Esses pesquisadores relatam os efeitos benéficos dos biocarvões nas propriedades físicas, químicas e biológicas do solo. A adição desse material ao solo promove acréscimos na CTC (SINGH et al., 2015), pH e disponibilidade de nutrientes para as plantas (EL-NAGGAR et al., 2015; 2016).
A diminuição da disponibilidade dos recursos hídricos e a deterioração da qualidade das águas superficiais e subterrâneas apontam para a necessidade de um aproveitamento racional desse recurso, com o mínimo de dano ao meio ambiente. De acordo com dados do Plano Estadual de Recursos Hídricos do Estado do Espírito Santo (PERH-ES, 2018) 78,4\% da água doce captada e 92,3\% da água doce consumida no Estado tem por finalidade a irrigação de culturas agrícolas.

Dessa forma, faz-se premente a necessidade de redução do consumo de água na agricultura e a identificação de alternativas viáveis para irrigação de culturas agrícolas, reduzindo assim a pressão sobre os mananciais hídricos do Estado. Dentre os resíduos agrícolas com potencial para utilização em substituição à água na irrigação destaca-se a água residuária de suinocultura (ARS). A ARS contém macro e micronutrientes que potencializam seu uso na fertirrigação de culturas agrícolas, como observado nos trabalhos de Moretti et al. (2017), Guidinelle (2019) e Sarto et al. (2019).

Diante do exposto, denota-se que a palha de café carbonizada (biocarvão) e ARS apresentam potencial na melhoria dos atributos físicos, químicos e biológicos do solo, que por sua vez podem contribuir significativamente para o desenvolvimento das plantas.

O objetivo geral do estudo foi avaliar o efeito de diferentes doses de biocarvão, proveniente da palha de café conilon, associado com a aplicação da água residuária de suinocultura, no 
desenvolvimento de plantas de café conilon.

\section{MATERIAL E MÉTODOS}

$\mathrm{O}$ experimento foi conduzido em casa de vegetação localizada no Instituto Federal do Espirito Santo (Ifes) - Campus de Alegre. O delineamento utilizado foi o inteiramente casualizado (DIC), num arranjo fatorial $5 \times 2$, com três repetições. Os tratamentos consistiram da combinação de cinco doses de biocarvão de palha de café conilon, equivalentes a $0,5,10,15$ e 20 t/ha, com duas águas de irrigação (água residuária de suinocultura-ARS e água bruta-AB).

Para a obtenção do biocarvão, a palha de café foi acondicionada em um reator de pirólise instalado no Centro de Ciências Agrárias e Engenharias da Universidade Federal do Espírito Santo (CCAE/UFES), sendo carbonizado a $350^{\circ} \mathrm{C}$. As características químicas do biocarvão são apresentadas na Tabela 1 . $\mathrm{O}$ biocarvão foi passado em peneira de $2 \mathrm{~mm}$ para homogeneizar o tamanho das partículas antes de sua utilização no experimento. A ARS foi coletada no setor de suinocultura do Ifes-Campus de Alegre, sendo originada do tratamento anaeróbio dos dejetos de suínos em biodigestor. A $\mathrm{AB}$ foi originária da água de abastecimento do Campus, sem tratamento. A análise da ARS foi realizada no laboratório de hidráulica e irrigação do CCAE/UFES e no laboratório Fullin (município de Linhares ES). Os resultados da análise são apresentados na Tabela 2.

O solo utilizado para o enchimento dos vasos foi um Latossolo vermelhoamarelo, coletado na profundidade 0-40 $\mathrm{cm}$, cujas características físicas e químicas, analisadas seguindo os protocolos descritos em Embrapa (2017), constam na Tabela 3.
Tabela 1: Caracterização química do biocarvão produzido com palha de café conilon a temperatura de $350^{\circ} \mathrm{C}$

\begin{tabular}{lcc}
\hline Atributo & Unidade & \\
\hline $\mathrm{pH}$ (em água) & - & 8,94 \\
$\mathrm{Cinzas} \mathrm{( \% )}$ & $\%$ & 13,66 \\
$\mathrm{CTC}$ & $\mathrm{cmol}_{\mathrm{c}} / \mathrm{dm}^{3}$ & 296,99 \\
$\mathrm{~K}$ & $\mathrm{~g} / \mathrm{dm}^{3}$ & 62,36 \\
$\mathrm{Ca}$ & $\mathrm{g} / \mathrm{dm}^{3}$ & 1,85 \\
$\mathrm{Mg}$ & $\mathrm{g} / \mathrm{dm}^{3}$ & 0,88 \\
$\mathrm{P}$ & $\mathrm{g} / \mathrm{dm}^{3}$ & 0,39 \\
$\mathrm{C}$ & $\%$ & 55,69 \\
$\mathrm{~N}$ & $\%$ & 3,52 \\
$\mathrm{H}$ & $\%$ & 4,36 \\
$\mathrm{O}$ & $\%$ & 36,43 \\
$\mathrm{H} / \mathrm{C}$ & - & 0,94 \\
$\mathrm{O} / \mathrm{C}$ & - & 0,49 \\
$\mathrm{C} / \mathrm{N}$ & - & 18,45 \\
\hline
\end{tabular}

Potássio (K), cálcio (Ca), magnésio (Mg) e fósforo (P) obtidos por extração total pela incineração das amostras em forno tipo mufla $\left(550^{\circ} \mathrm{C} ; 4 \mathrm{~h}\right)$, com a solubilização das cinzas em $\mathrm{HCl} 0,5 \mathrm{~mol} / \mathrm{L}$ e dosagem por espectrofotometria de absorção atômica; K, Ca e Mg solúveis, obtidos por extração em solução de $\mathrm{HCl}$ 0,1 mol/L e dosagem por espectrofotometria de absorção atômica; carbono (C) obtido por oxidação da matéria orgânica por via úmida, utilizando-se solução de $\mathrm{K}_{2} \mathrm{Cr}_{2} \mathrm{O}_{7}$ em meio ácido; nitrogênio $(\mathrm{N})$ pelo método de Kjeldahl; teores totais de $\mathrm{C}, \mathrm{H}, \mathrm{N}$ e $\mathrm{O}$ obtidos em analisador elementar

Fonte: Dados da pesquisa.

Tabela 2: Caracterização química da água residuária de suinocultura (ARS)

\begin{tabular}{|c|c|c|}
\hline Parâmetro Analisado & Unidade & \\
\hline Condutividade Elétrica (C.E.) & $\mathrm{dS} / \mathrm{m}$ & 5,16 \\
\hline $\mathrm{pH}$ & - & 7,9 \\
\hline $\begin{array}{l}\text { Razão de Adsorção Sódio } \\
\text { (RAS) }\end{array}$ & - & 1,6 \\
\hline Ferro total $(\mathrm{Fe})$ & $\mathrm{mg} / \mathrm{L}$ & 0,52 \\
\hline Sódio $(\mathrm{Na})$ & $\mathrm{mg} / \mathrm{L}$ & 50,0 \\
\hline Cloro (Cl) & $\mathrm{mg} / \mathrm{L}$ & $<0,15$ \\
\hline Zinco (Zn) & $\mathrm{mg} / \mathrm{L}$ & 0,11 \\
\hline Cobre $(\mathrm{Cu})$ & $\mathrm{mg} / \mathrm{L}$ & $<0,05$ \\
\hline Sulfato $\left(\mathrm{SO}_{4}{ }^{2-}\right)$ & $\mathrm{mg} / \mathrm{L}$ & $<5,0$ \\
\hline Fósforo $\left(\mathrm{PO}_{4}^{3-}\right)$ & $\mathrm{mg} / \mathrm{L}$ & 61,87 \\
\hline Potássio (K) & $\mathrm{mg} / \mathrm{L}$ & 210,0 \\
\hline Cálcio (Ca) & $\mathrm{mg} / \mathrm{L}$ & 54,4 \\
\hline Magnésio (Mg) & $\mathrm{mg} / \mathrm{L}$ & 12,0 \\
\hline Boro (B) & $\mathrm{mg} / \mathrm{L}$ & 0,42 \\
\hline Manganês (Mn) & $\mathrm{mg} / \mathrm{L}$ & 0,25 \\
\hline Nitrogênio (N) $\left(\mathrm{NO}_{3}{ }^{-}\right)$ & $\mathrm{mg} / \mathrm{L}$ & 2,3 \\
\hline Nitrogênio (N) $\left(\mathrm{NH}_{4}^{+}\right)$ & $\mathrm{mg} / \mathrm{L}$ & 511,0 \\
\hline
\end{tabular}


Tabela 3: Caracterização física e química do solo utilizado no experimento

\begin{tabular}{lcl}
\hline Atributo & Unidade & \\
\hline Argila & $\%$ & 49,0 \\
Areia total & $\%$ & 46,0 \\
Silte & $\%$ & 5,0 \\
$\mathrm{pH}$ & & 5,29 \\
$\mathrm{Ca}^{2+}$ & $\mathrm{cmol}_{\mathrm{c}} / \mathrm{dm}^{3}$ & 0,87 \\
$\mathrm{Mg}^{2+}$ & $\mathrm{Cmol}_{\mathrm{c}} / \mathrm{dm}^{3}$ & 1,17 \\
$\mathrm{P}\left(\mathrm{Mehlich}^{3} 1\right)$ & $\mathrm{mg} / \mathrm{dm}^{3}$ & 8,03 \\
$\mathrm{~K}^{+}$ & $\mathrm{mg} / \mathrm{dm}^{3}$ & 36 \\
$\mathrm{Na}^{+}$ & $\mathrm{mg} / \mathrm{dm}^{3}$ & 0,0 \\
$\mathrm{Al}^{3+}$ & $\mathrm{cmol}_{\mathrm{c}} / \mathrm{dm}^{3}$ & 0,1 \\
$\mathrm{H}^{+} \mathrm{Al}^{3+}$ & $\mathrm{cmol} / \mathrm{dm}^{3}$ & 2,64 \\
$\mathrm{~T}$ & $\mathrm{cmol} / \mathrm{dm}^{3}$ & 4,77 \\
$\mathrm{~m}$ & $\%$ & 4,48 \\
$\mathrm{~V}$ & $\%$ & 44,66 \\
\multicolumn{2}{c}{ Fonte: Dados da pesquisa }
\end{tabular}

Após coletado, o solo foi seco ao ar e peneirado em peneira com malha de 2 $\mathrm{mm}$ a fim de homogeneização, sendo posteriormente incubado por 21 dias com as doses de biocarvão e calcário para a correção da acidez do solo. Foram utilizadas plantas de café conilon (Coffea canephora Pierre ex A. Froehner) do cultivar EMCAPER 8151 - Robusta Tropical, produzidas em viveiro e posteriormente transplantadas para o vaso.

Foi cultivada uma planta por vaso (capacidade de 10 litros), preenchidos com os tratamentos referentes ao solo incubado com biocarvão. No momento do transplantio foi realizada a adubação para o suprimento de $\mathrm{N}$ e $\mathrm{P}$, utilizando as recomendações de Oliveira et al. (1991), para a adubação em ambientes controlados. Diariamente foi realizada a irrigação das plantas com ARS ou AB. O controle da irrigação foi efetuado por meio da pesagem diária dos vasos, de forma a manter a umidade do solo em $50 \%$ do volume total de poros.

O experimento foi conduzido por 150 dias, findo os quais foram avaliados os seguintes parâmetros morfoagronômicos de desenvolvimento do cafeeiro: altura de planta - AT (cm); diâmetro do caule - DC (mm); número de ramos plagiotrópicos NRP; comprimento do maior ramo plagiotrópico - CMRP; número total de nós - NN; massa da matéria fresca da parte aérea - MFPA (g); massa da matéria fresca de raiz - MFR (g); massa da matéria fresca total - MFT (g); massa da matéria seca da parte aérea - MSPA (g); massa da matéria seca de raiz - MSR (g) e massa da matéria seca total - MST (g). A avaliação da produção de massa da matéria seca consistiu na separação da parte aérea e raiz, com posterior lavagem para remoção de resíduos, sua disposição em sacos de papel e secagem em estufa de circulação forçada de ar a $70^{\circ} \mathrm{C}$, até peso constante. Após o processo de secagem, procedeu-se a pesagem da MSPA e MSR em balança analítica. A massa da matéria seca total foi obtida pela soma dos valores de MSPA + MSR.

Para a avaliação do efeito das doses de biocarvão sobre os diferentes atributos avaliados nas plantas de café, foi realizada a análise de regressão, sendo os modelos escolhidos com base na significância dos coeficientes de regressão, utilizando-se o teste $\mathrm{t}$ de Student ao nível de $5 \%$ de probabilidade e o coeficiente de determinação $\left(\mathrm{R}^{2}\right)$. Possíveis diferenças entre as águas de irrigação (ARS e AB) e o desdobramento da interação entre fonte água e dose de biocarvão, foram avaliadas pelo teste de $\mathrm{F}$ da análise de variância ao nível de 5\% de probabilidade. As análises estatísticas foram efetuadas por meio do aplicativo computacional SISVAR (FERREIRA, 2000).

\section{RESULTADOS E DISCUSSÃO}

O desdobramento da interação entre águas de irrigação e doses de biocarvão foi significativo $(\mathrm{p} \leq 0,05)$ para os parametros DC, NTN, MFPA, MFT e MST (Tabela 4). Os parâmetros ALT, NRP, CMRP, MFR, MSR e MSPA apresentaram ausência de interação significativa para o desdobramento da interação, sendo apresentados os resultados das médias gerais de todas as doses (Tabela 5).

Na tabela 4 é possivél identificar que todos os tratamentos submetidos à dose de 15 t/ha de biocarvão e irrigados 
com ARS apresentaram melhor desenvolvimento, com respostas positivas para os seguintes parâmetros: DC, NN, MFPA, MFT, e MST. Uma hipótese para esse resultado é o efeito sinergico entre a ARS e o biocarvão relatado por Cunha (2018), ao trabalhar com aplicação de ARS e diferentes doses de biocarvão no desenvolvimento inicial da cultura do milho em casa de vegetação. De acordo com Petter \& Madari (2012), o biocarvão potencializa o P e K fornecido pela ARS. Os autores creditam esse efeito a presença de sítios eletroquímicos na superfície do biocarvão capazes de reter nutrientes, potencializando o desenvolvimento vegetativo das plantas.

Tabela 4: Efeitos da irrigação com água residuária de suinocultura (ARS) e água bruta (AB) em atributos morfoagronômicos de plantas de café conilon cultivadas em vasos com aplicação de diferentes doses de biocarvão

\begin{tabular}{cccccc}
\hline \multirow{2}{*}{ Água } & \multicolumn{5}{c}{ Doses de biocarvão (t/ha) } \\
\cline { 2 - 6 } & 0 & 5 & 10 & 15 & 20 \\
ARS & $8,0 \mathrm{a}$ & $7,8 \mathrm{a}$ & $7,8 \mathrm{a}$ & $9,3 \mathrm{a}$ & $7,6 \mathrm{a}$ \\
AB & $8,0 \mathrm{a}$ & $6,7 \mathrm{a}$ & $7,4 \mathrm{a}$ & $6,9 \mathrm{~b}$ & $7,5 \mathrm{a}$ \\
& & $\mathbf{N N}$ \\
ARS & $17,7 \mathrm{a}$ & $22,0 \mathrm{a}$ & $17,3 \mathrm{a}$ & $38,0 \mathrm{a}$ & $24,0 \mathrm{a}$ \\
AB & $18,7 \mathrm{a}$ & $20,0 \mathrm{a}$ & $15,3 \mathrm{a}$ & $22,0 \mathrm{~b}$ & $25,7 \mathrm{a}$ \\
& & \multicolumn{5}{c}{ MFPA (g) } \\
ARS & $36,0 \mathrm{a}$ & $57,4 \mathrm{a}$ & $39,8 \mathrm{a}$ & $90,6 \mathrm{a}$ & $43,7 \mathrm{a}$ \\
$\mathrm{AB}$ & $46,8 \mathrm{a}$ & $37,6 \mathrm{a}$ & $37,5 \mathrm{a}$ & $36,2 \mathrm{~b}$ & $34,2 \mathrm{a}$ \\
& & \multicolumn{5}{c}{ MFT (g) } \\
ARS & $50,1 \mathrm{a}$ & $71,3 \mathrm{a}$ & $48,7 \mathrm{a}$ & $116,09 \mathrm{a}$ & $61,8 \mathrm{a}$ \\
AB & $66,1 \mathrm{a}$ & $54,6 \mathrm{a}$ & $63,7 \mathrm{a}$ & $59,0 \mathrm{~b}$ & $62,1 \mathrm{a}$ \\
& & \multicolumn{5}{c}{ MST (g) } \\
ARS & $23,0 \mathrm{a}$ & $33,7 \mathrm{a}$ & $20,9 \mathrm{a}$ & $40,9 \mathrm{a}$ & $24,7 \mathrm{a}$ \\
AB & $26,3 \mathrm{a}$ & $22,4 \mathrm{~b}$ & $23,2 \mathrm{a}$ & $21,6 \mathrm{~b}$ & $23,7 \mathrm{a}$ \\
\hline
\end{tabular}

As médias seguidas pelas mesmas letras minúsculas na coluna não diferem entre si pelo teste $\mathrm{F}$ a nível de $5 \%$ de probabilidade. DC: diâmetro do caule; NN: número total de nós; MFPA: massa da matéria fresca da parte aérea; MFT: massa da matéria fresca total; MSR: massa da matéria seca de raiz; MST: massa da matéria seca total. Fonte: Dados da pesquisa.

A irrigação com a ARS proporcionou um bom desenvolvimento das plantas, fato também observado por Batista et al. (2014) que relataram efeito positivo da ARS na altura de plantas de eucalipto. O mesmo foi observado no presente trabalho com plantas de café. Além disso, a ARS proporcionou um maior desenvolvimento do NRP e MSPA (Tabela 5). Segundo Piava et al. (2009), características relacionadas a produção de biomassa, como a MSPA, são importantes na avaliação do desenvolvimento de espécies vegetais, demonstrando seu potencial vegetativo, complementando os resultados de altura total de planta. Esse resultado pode ser considerado de grande importância, pois segundo Ferrão et al. (2008) entre as características que mais se relacionam com a produtividade da cultura do café conilon está altura de plantas e o número de ramos plagiotrópicos.

O bom desenvolvimento do cafeeiro pode estar relacionado com as concentrações de macronutrientes contidos na ARS (61,87mg/L de P; $210 \mathrm{mg} / \mathrm{L}$ de $\mathrm{K} ; 2,3 \mathrm{mg} / \mathrm{L}$ de $\mathrm{N}-\mathrm{NO}_{3}{ }^{-}$e; 511,00 mg/L de N-NH${ }_{4}^{+}$). 
Tabela 5: Médias gerais do efeito da irrigação com água residuária de suinocultura (ARS) e água bruta (AB) em atributos morfoagronômicos de plantas de café conilon cultivadas em vasos com aplicação de diferentes doses de biocarvão

\begin{tabular}{|c|c|}
\hline Água & Parâmetros \\
\hline \multicolumn{2}{|c|}{ ALT } \\
\hline ARS & $43,24 a$ \\
\hline $\mathrm{AB}$ & $38,95 b$ \\
\hline \multicolumn{2}{|c|}{ NRP } \\
\hline ARS & $4,73 a$ \\
\hline $\mathrm{AB}$ & $3,33 b$ \\
\hline \multicolumn{2}{|c|}{ CMRP } \\
\hline ARS & $13,10 a$ \\
\hline $\mathrm{AB}$ & $10,77 \mathrm{a}$ \\
\hline \multicolumn{2}{|c|}{ MFR } \\
\hline ARS & $16,05 a$ \\
\hline $\mathrm{AB}$ & $20,84 a$ \\
\hline \multicolumn{2}{|c|}{ MSR } \\
\hline ARS & $6,04 a$ \\
\hline $\mathrm{AB}$ & $7,23 a$ \\
\hline \multicolumn{2}{|c|}{ MSPA } \\
\hline ARS & $22,61 a$ \\
\hline $\mathrm{AB}$ & $16,23 b$ \\
\hline
\end{tabular}

As médias seguidas pelas mesmas letras minúsculas na coluna não diferem entre si pelo teste $\mathrm{F}$ a nível de 5\% de probabilidade. AT: altura de planta; NRP: número de ramos plagiotrópicos; CMRP: comprimento do maior ramo plagiotrópico; MFR: massa da matéria fresca de raiz; MSR: massa da matéria seca de raiz; MSPA: massa da matéria seca da parte aérea.

Fonte: Dados da pesquisa.

(Tabela 2). Do total de N, P e K contidos na ARS, aproximadamente $67 \%$ do N, 33\% do P e $100 \%$ do K encontram-se na forma mineral, prontamente disponíveis para as plantas no primeiro ano de aplicação (GOMES FILHO et al., 2001).

A utilização de águas residuárias na agricultura é importante tanto para o suprimento de nutrientes quanto para o atendimento da demanda hídrica das culturas (BARROS et al., 2015). Quando essas águas são aplicadas na superfície do solo, ocorrem processos de depuração de natureza física, química e biológica no sistema solo-planta-água (LO MONACO, 2005). Assim, as plantas absorvem dessas águas macro e micronutrientes necessários para o seu desenvolvimento, evitando acúmulos no solo (MATOS et al., 2003). No entanto, para alcançar bons resultados agronômicos e ambientais com o uso agrícola da ARS faz-se necessário realizar frequentes análises químicas para caracterização do solo e da ARS, evitando degradação ambiental, por se tratar de um resíduo com potencial poluidor (CÔRREA et al., 2011).

Apesar dos resultados significativos para a aplicação de ARS na dose de 15 t/ha de biocarvão, para todas as variáveis de desenvolvimento do cafeeiro avaliadas, exceto número total de nós, não houve efeito das diferentes doses de biocarvão de palha de café conilon, razão pela qual são apresentadas as equações de regressão dos efeitos da variável quantitativa no formato de tabela (Tabela 6). 
Tabela 6: Significância dos modelos de regressão linear e quadrática para efeito das diferentes doses de biocarvão dentro das diferentes águas de irrigação (ARS e AB) nas características morfoagronômicas do cafeeiro conilon

\begin{tabular}{|c|c|c|c|c|c|}
\hline Variável & Água & Equação & Valor de p & $\mathbf{R}^{2}$ & Significância \\
\hline \multirow{4}{*}{ AT } & \multirow{2}{*}{ ARS } & $0,006 x+43,173$ & 0,9640 & 0,003 & ns \\
\hline & & $0,003 x^{2}-0,0580 x+43,335$ & 0,8969 & 0,03 & ns \\
\hline & \multirow{2}{*}{$\mathrm{AB}$} & $0,008 x+38,873$ & 0,9646 & 0,04 & ns \\
\hline & & $0,015 x^{2}-0,296 x+39,635$ & 0,6183 & 0,51 & ns \\
\hline \multirow{4}{*}{ DC } & \multirow{2}{*}{ ARS } & $0,008 x+7,988$ & 0,7293 & 0,09 & ns \\
\hline & & $-0,004 x^{2}+0,108 x+7,738$ & 0,2643 & 0,11 & ns \\
\hline & \multirow{2}{*}{$\mathrm{AB}$} & $-0,012 x+7,478$ & 0,6863 & 0,03 & ns \\
\hline & & $0,008 x^{2}-0,177 x+7,891$ & 0,1305 & 0,56 & ns \\
\hline \multirow{2}{*}{ NRP } & \multirow{2}{*}{$\mathrm{ARS} / \mathrm{AB}^{*}$} & $0,010 x+3,93$ & 0,8373 & 0,13 & ns \\
\hline & & $-0,001 x^{2}+0,038 x+3,86$ & 0,8622 & 0,22 & ns \\
\hline \multirow{2}{*}{ CMRP } & \multirow{2}{*}{$\mathrm{ARS} / \mathrm{AB}^{*}$} & $0,019 x+11,736$ & 0,8905 & 0,006 & ns \\
\hline & & $0,009 x^{2}-0,160 x+12,187$ & 0,7114 & 0,05 & ns \\
\hline \multirow{2}{*}{ NN } & \multirow{2}{*}{$\mathrm{ARS} / \mathrm{AB}^{*}$} & $0,446 x+17,600$ & 0,0029 & 0,41 & $*$ \\
\hline & & $0,006 x^{2}+0,313 x+17,933$ & 0,7674 & 0,41 & ns \\
\hline \multirow{2}{*}{ MFR } & \multirow{2}{*}{ ARS/AB* } & $0,244 x+16,003$ & 0,2048 & 0,33 & ns \\
\hline & & $-0,012 x^{2}+0,485 x+15,401$ & 0,7063 & 0,36 & ns \\
\hline \multirow{4}{*}{ MFPA } & \multirow{2}{*}{ ARS } & $0,974 x+43,780$ & 0,1582 & 0,11 & ns \\
\hline & & $-0,195 x^{2}+4,880 x+34,014$ & 0,1006 & 0,28 & ns \\
\hline & \multirow{2}{*}{$\mathrm{AB}$} & $-0,173 x+42,002$ & 0,6681 & 0,09 & ns \\
\hline & & $0,088 x^{2}-1,946 x+46,433$ & 0,2122 & 0,92 & ns \\
\hline \multirow{2}{*}{ MFT } & \multirow{2}{*}{$\mathrm{ARS} / \mathrm{AB}^{*}$} & $0,644 x+58,896$ & 0,1558 & 0,16 & ns \\
\hline & & $-0,065 x^{2}+1,952 x+55,625$ & 0,3865 & 0,21 & ns \\
\hline \multirow{2}{*}{ MSR } & \multirow{2}{*}{$\mathrm{ARS} / \mathrm{AB}^{*}$} & $0,090 x+5,737$ & 0,3231 & 0,92 & ns \\
\hline & & $0,001 x^{2}+0,056 x+5,821$ & 0,9127 & 0,93 & ns \\
\hline \multirow{4}{*}{ MSPA } & \multirow{2}{*}{ ARS } & $0,007 x+22,538$ & 0,9814 & 0,001 & ns \\
\hline & & $-0,0849 x^{2}+1,706 x+18,292$ & 0,1321 & 0,23 & ns \\
\hline & \multirow{2}{*}{$\mathrm{AB}$} & $-0,094 x+17,181$ & 0,5913 & 0,09 & ns \\
\hline & & $0,048 x^{2}-1,061 x+19,598$ & 0,1230 & 0,97 & ns \\
\hline \multirow{4}{*}{ MST (g) } & \multirow{2}{*}{ ARS } & $0,214 x+26,512$ & 0,4725 & 0,04 & ns \\
\hline & & $-0,060 x^{2}+1,423 x+23,488$ & 0,2404 & 0,15 & ns \\
\hline & \multirow{2}{*}{$\mathrm{AB}$} & $-0,012 x+24,682$ & 0,5232 & 0,28 & ns \\
\hline & & $0,027 x^{2}-0,666 x+26,047$ & 0,3969 & 0,79 & ns \\
\hline
\end{tabular}

Significativo pelo teste $\mathrm{F}$ a $5 \%$ de probabilidade. *Ausência de diferença significativa entre as diferentes fontes de água de irrigação (Água residuária de suinocultura - ARS e Água bruta - AB).

Fonte: Dados da pesquisa.

A ausência de respostas significativas da aplicação das doses de biocarvão no desenvolvimento do café conilon pode estar relacionada a dois fatores: i) os efeitos do biocarvão nas propriedades do solo e desenvolvimento do cafeeiro, em função de sua natureza orgânica, demandam estudos conduzidos por período maior do que o avaliado no presente trabalho; ii) o cafeeiro, por ser uma cultura de ciclo perene, responde mais lentamente às mudanças nos sistemas de manejo.
Resultados semelhantes foram encontrados por Liang et al. (2014), onde não foram encontradas diferenças significativas na produtividade de milho e trigo com a aplicação de carvão pirogênico (30, 60 ou 90 t/ha), durante três anos de avaliação.

Segundo Novak et al. (2009), experimentos de curto prazo, assim como este apresentado, podem ser úteis para determinar os efeitos iniciais do biocarvão no solo, como por exemplo as mudanças na capacidade de retenção de água. 
Todavia, mudanças significativas relacionadas a consolidação, intemperismo das partículas e lavagem de cinzas para disponibilização dos elementos solúveis presentes no biocarvão são improváveis de serem encontradas em estudos de curta duração (BURRELL at al., 2016). De acordo com Liu et al. (2013) experimentos de longo prazo têm maior probabilidade de produzirem resultados que representam efeitos positivos no solo, o que resultaria em um maior desenvolvimento de plantas. Os resultados encontrados por Laird et al. (2010), corroboram com essa afirmação. Os autores afirmam que, de forma geral, após 500 dias do uso do biocarvão os resultados positivos na qualidade do solo foram muito mais evidentes.

Outra circunstância a ser destacada é a temperatura de carbonização do biocarvão. Schimmelpfennig e Glaser (2011) relatam que um aumento na temperatura da pirólise até aproximadamente $750^{\circ} \mathrm{C}$ proporciona um aumento na porosidade do biocarvão, o que favorece as reações químicas e física no solo. Neste estudo, a temperatura de carbonização foi de $350^{\circ} \mathrm{C}$, o que pode prolongar o tempo de reação do biocarvão no solo.

Para o parâmetro número total de nós $(\mathrm{NN})$ foi observado efeito significativo das doses de biocarvão no desdobramento das médias das duas fontes de água de irrigação (Figura 1). Estudos que avaliam o efeito da aplicação de biocarvão e ARS no desenvolvimento do cafeeiro conilon são recentes não existindo, até o momento, relatos de publicações dessa natureza. Segundo Jaeggi et al. (2019), plantas de café conilon fertilizadas com adubo convencional apresentam efeito indireto altamente positivo para produção de nós, ou seja, uma determinada concentração de nutrientes incrementa a produção total de nós pelo cafeeiro. De posse dessa informação, uma hipótese para os resultados de $\mathrm{NN}$ do presente trabalho pode ser a solubilização e disponibilização de nutrientes adicionados o solo pelas doses de biocarvão, aumentando a absorção de nutrientes pelas plantas, resultando no incremento do NN.

Figura 1: Efeito de diferentes doses de biocarvão e água de irrigação no número total de nós de plantas de café conilon.

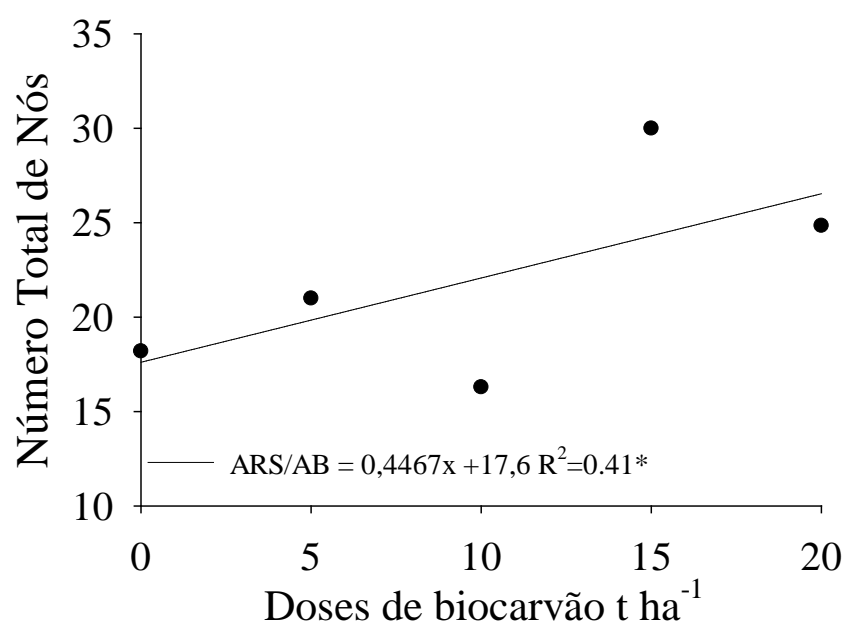

*significativo pelo teste $\mathrm{F}$ a $5 \%$ de probabilidade Fonte: Os autores.

Os resultados apresentados indicam que as principais respostas significativas no desenvolvimento das plantas de café conilon ocorreram pela aplicação da ARS. Esses resultados certamente estão associados à presença de nutrientes na ARS, necessários ao desenvolvimento das plantas de café.

\section{CONCLUSÕES}

As doses de biocarvão não influenciaram o desenvolvimento do cafeeiro conilon até os 150 dias de avaliação.

A irrigação com água residuária de suinocultura promoveu os melhores resultados no desenvolvimento do cafeeiro conilon, com destaque para os parâmetros altura de planta, número de ramos plagiotrópicos e massa da matéria seca da parte aérea. 
A combinação da dose de 15 t/ha de biocarvão com água residuária de suinocultura apresentou os melhores resultados para diâmetro do caule, número total de nós, massa da matéria fresca da parte aérea, massa da matéria fresca total, indicando um efeito sinérgico.

\section{AGRADECIMENTOS}

Ao Ifes pela concessão da bolsa de iniciação científica.

\section{REFERÊNCIAS}

BARROS, H. M. M.; BARROS, M. K. L. V.; DE PÁDUA SOUZA, L.; CHICÓ, L. R.; BAROSI, K. X. L. Reúso de água na agricultura. Revista Verde de

Agroecologia e Desenvolvimento

Sustentável, v.10, n.5, p.3, 2015.

BATISTA, R. O.; MARTINEZ, M. A.; PAIVA, H. N. D.; BATISTA, R. O.; CECON, P. R. O efeito da água residuária da suinocultura no desenvolvimento e qualidade de mudas de Eucalyptus urophylla. Ciência Florestal, v.24, n.1, p. 127-135, 2014.

BURRELL, L. D.; ZEHETNER, F.; RAMPAZZO, N.; WIMMER, B.; SOJA, G. Long-term effects of biochar on soil physical properties. Geoderma, v.282, p. 96-102, 2016.

CÔRREA, J. C.; NICOLOSO R. S.; MENEZES, J. F. S.; BENITES, V. M.

Critérios técnicos para recomendação de biofertilizante de origem animal em sistema de produção agrícolas e florestais. Concórdia/SC: Embrapa Suínos e Aves, 2011.

CUNHA, E. G. Influência de biocarvão e água residuária da suinocultura nos atributos do solo, no desenvolvimento inicial e na nutrição mineral do milho.
Dissertação (Mestrado em Agroecologia) Programa de Pós-Graduação em Agroecologia do Instituto Federal do Espírito Santo - IFES, Campus de Alegre, ES, 2018.

EL-NAGGAR, A.; LEE, S. S.;

RINKLEBE, J.; FAROOQ, M.; SONG, H.; SARMAH, A. K.; OK, Y. S. Biochar application to low fertility soils: a review of current status, and future prospects.

Geoderma v.337, p.536-554, 2019.

EL-NAGGAR, A. H.; AHMAD, M.; ALFARAJ, A.; AL-OMRAN, A. Conocarpus biochar induces changes in soil nutrient availability and tomato growth under saline irrigation. Pedosphere, v. 26, n. 1, p. 2738, 2016.

EL-NAGGAR, A. H.; USMAN, A. R. A.; AL-OMRAN, A.; OK, Y. S.; AHMAD, M.; AL WABEL, M. I. Carbon mineralization and nutrient availability in calcareous sandy soils amendes with woody waste biochar.

Chemosphere, v.138, p. 67-73, 2015.

EMPRESA BRASILEIRA DE PESQUISA AGROPECUÁRIA - EMBRAPA. Manual de métodos de análise de solo. 3 . ed. Brasília, DF. 2017, 575p.

FERRÃO, R. G.; CRUZ, C. D.; FERREIRA, A.; CECON, P. R.; FERRÃO, M. A. G.; FONSECA, A. F. A. D.; ...

SILVA, M. F. D. Parâmetros genéticos em café conilon. Pesquisa Agropecuária Brasileira, v.43, n.1, p.61-69, 2008.

FERREIRA, D. F. Análises estatísticas por meio do Sisvar para Windows 4. 0. In: REUNIÃO ANUAL DA REGIÃO BRASILEIRA DA SOCIEDADE INTERNACIONAL DE BIOMETRIA, 45. 2000, São Carlos. Anais... São Carlos, SP: UFSCAR, p. 255-258. 2000. 
FONSECA, A. A.; SANTOS, D. A.; PASSOS, R. R.; ANDRADE, F. V.; RANGEL, O. J. P. Phosphorus availability and grass growth in biochar-modified acid soil: a study excluding the effects of soil $\mathrm{pH}$. Soil Use and Management, v.36, n.4, p.714-725, 2020.

GOMES FILHO, R. R.; MATOS, A. T.; SILVA, D. D. MARTINEZ, H E.P. Remoção de carga orgânica e produtividade da aveia forrageira em cultivo hidropônico com águas residuárias da suinocultura. Revista Brasileira de Engenharia Agrícola e Ambiental, Campina Grande, v.5, n.1, p. 131-134. 2001.

GUIDINELLE, R. B. Água residuária de suinocultura e sistema plantio direto no desenvolvimento do milho para produção de silagem. Dissertação (Mestrado em Agroecologia) - Programa de Pós-Graduação em Agroecologia do Instituto Federal do Espírito Santo - IFES, Campus de Alegre, ES, 2019.

JAEGGI, M. E. P.C.; COELHO, F. C.; PEREIRA, I. M.; ZACARIAS, A. J.; DE AMARAL GRAVINA, G.; LIMA, W. L.; CARMO-PARAJARA, M. Path Analysis of vegetative characteristics in conilon coffee production consortiated with green fertilizers in tropical climate. Journal of Experimental Agriculture International, v.40, n.2, p.1-11, 2019.

KOURAA, A.; FETHI, F.; FAHDE, A.; LAHLOU, A.; QUAZZANI, N. Reuse of urban wastewater treated by a combined stabilization pond system in Benslimane (Morocco). Urban Water, v.4, p.373-378, 2002.

LAIRD, D. A.; FLEMING, P.; DAVIS, D. D.; HORTON, R.; WANG, B.; KARLEN, D. L. Impact of biochar amendments on the quality of a typical Midwestern agricultural soil. Geoderma, v.158, n.3-4, p. 443-449, 2010.
LIANG, F.; LI, G.; LIN, Q.; ZHAO, X. Crop yield and soil properties in the first 3 years after biochar application to a calcareous soil. Journal of Integrative Agriculture, v 13, p. 525-532, 2014.

LIU, X.; ZHANG, A.; JI, C.; JOSEPH, S.; BIAN, R.; LI, L.; PAN, G.; PAZFERREIRO, J. Biochar's effect on crop productivity and the dependence on experimental conditions - a metaanalysis of literature data. Plant Soil, v.373, p. 583594, 2013.

\section{LO MONACO, P. A. Fertirrigação do} cafeeiro com águas residuárias da lavagem e descascamento de seus frutos. 2005. 96 f. Tese (Doutorado) Universidade Federal de Viçosa, Viçosa, 2005.

MAROUŠEK, J.; STRUNECKÝ, O.; STEHEL, V. Biochar farming: defining economically perspective applications. Clean Technologies and Environmental Policy, v.21, p. 1389-1395, 2019.

MATOS, A.T. de; PINTO, A. B.; PEREIRA, O. G.; SOARES, A.A.; LO MONACO, P. A. Produtividade de forrageiras utilizadas em rampas de tratamento de águas residuárias da lavagem e despolpa dos frutos do cafeeiro. Revista Brasileira de Engenharia Agrícola e Ambiental, v.7, n.1, p.154-158, 2003.

MESA-PÉREZ, J. M.; CORTEZ, L. A.; ROCHA, J. D.; GOMEZ, E. O. Pirólise rápida em leito fluidizado: uma opção para transformar biomassa em energia limpa.

Revista Analytica, São Paulo, v.4, p.3236, 2003.

MORETTI, S. M. L.; BERTONCINI, E. I.; ABREU-JUNIOR, C. H. Carbon mineralization in soils irrigated with treated swine wastewater. Journal of 
Agricultural Science, v.9, n.3, p. 19-29, 2017.

NOVAK, J. M.; LIMA, I.; XING, B.; GASKIN, J. W.; STEINER, C.; DAS, K. C.; AHMEDNA, M.; REHRAH, D.; WATTS, D. W.; BUSSCHER, W. J.; SCHOMBERG, H. Characterization of designer biochar produced at different temperatures and their effects on a loamy sand. Annals of Environmental Science, v.3, p.195-206, 2009.

OLIVEIRA, A. J. de; GARRIDO, W. E.; ARAUJO, J. D. de; LOURENÇO, S. Métodos de pesquisa em fertilidade do solo. Brasília, DF: EMBRAPA-SEA, 1991. $392 \mathrm{p}$.

OLIVEIRA, F. R.; PATEL, A. K.; JAISI, D. P.; ADHIKARI, S.; LU, H.; KHANAL, S. K. Environmental application of biochar: current status and perspectives.

Bioresource technology, v.246, p. 110122, 2017.

PAIVA, A. V.; POGGIANI, F.; GONÇALVES, J. L. M.; FERRAZ, A. V. Crescimento de mudas de espécies arbóreas nativas, adubadas com diferentes doses de lodo de esgoto seco e com fertilização mineral. Scientia Forestalis, v. 37, n. 84, p. 499-511, 2009.

PELISSARI, R. A. Z.; SAMPAIO, S. C.; GOMES, S. D.; CREPALLI, M. da S. Lodo têxtil e água residuária da suinocultura na produção de mudas de Eucalyptus grandis. (W, Hill exMaiden).

Revista Engenharia Agrícola, v. 29, n. 2, p. 288-300, 2009.

PETTER, F. A.; MADARI, B. E. Biochar: agronomic and environmental potential in Brazilian savannah soils. Revista Brasileira de Engenharia Agrícola e Ambiental, v. 16, n.7, p. 761-768, 2012.
PERH/ES - PLANO ESTADUAL DE RECURSOS HÍDRICOS DO ESTADO. 2018 Disponível em https://perh.es.gov.br/Media/perh/Arquivos \%20Biblioteca/Cadernos/Caderno_Diagno stico_PERHES.pdf.

SARTO, J. R. W.; NERES, M. A.; SUNAHARA, S. M. M.; NATH, C. D.; SARTO, M. V. M. Chemical composition of swine wastewater, soil, and tifton 85 after 8 years of application. Revista Caatinga, v.32, n.1, p.259-269, 2019.

SCHIMMELPFENNIG, S; GLASER, B. One step forward toward characterization: some important material properties to distinguish biochars. Journal of Environmental Quality, v.41, p. 10011013. 2011.

SHACKLEY, S.; CARTER, S.; KNOWLES, T.; MIDDELINK, E.; HAEFELE, S.; CROSS, A.; HASZELDINE, S. Sustainable gasification biochar systems? a case study of rice-husk gasification in Cambodia, Part 1: context, chemical properties, environmental and health and safety issues. Energy Policy, v.42, p. 49-58, 2012

SINGH, R.; BABU, J.N.; KUMAR, R.; SRIVASTAVA, P.; SINGH, P.; RAGHUBANSHI, A. S. Multifaceted application of crop residue biochar as a tool for sustainable agriculture: an ecological perspective. Ecological Engineering, v.77, p.324-347, 2015. 